Research Article

\title{
Black Hole Entropy from Indistinguishable Quantum Geometric Excitations
}

\author{
Abhishek Majhi \\ The Institute of Mathematical Sciences, CIT Campus, 4th Cross St., Taramani, Chennai, Tamil Nadu, India \\ Correspondence should be addressed to Abhishek Majhi; abhishek.majhi@gmail.com
}

Received 4 August 2016; Accepted 9 October 2016

Academic Editor: Seyed H. Hendi

Copyright (C) 2016 Abhishek Majhi. This is an open access article distributed under the Creative Commons Attribution License, which permits unrestricted use, distribution, and reproduction in any medium, provided the original work is properly cited. The publication of this article was funded by SCOAP ${ }^{3}$.

In loop quantum gravity the quantum geometry of a black hole horizon consists of discrete nonperturbative quantum geometric excitations (or punctures) labeled by spins, which are responsible for the quantum area of the horizon. If these punctures are compared to a gas of particles, then the spins associated with the punctures can be viewed as single puncture area levels analogous to single particle energy levels. Consequently, if we assume these punctures to be indistinguishable, the microstate count for the horizon resembles that of Bose-Einstein counting formula for gas of particles. For the Bekenstein-Hawking area law to follow from the entropy calculation in the large area limit, the Barbero-Immirzi parameter $(\gamma)$ approximately takes a constant value. As a by-product, we are able to speculate the state counting formula for the SU(2) quantum Chern-Simons theory coupled to indistinguishable sources in the weak coupling limit.

\section{Introduction}

One of the prime achievements of canonical quantum gravity, more specifically loop quantum gravity (LQG), is the provision of a description of microstates of equilibrium black hole horizon, modeled as quantum isolated horizon (IH) $[1,2]$, leading to an $a b$ initio quantum statistical derivation of entropy from first principles. The quantum geometry of a cross section of an $\mathrm{IH}$ is depicted as a topological 2-sphere with quantum degrees of freedom at localized points called punctures. These punctures are the quantum geometry and each puncture is associated with a quantum number $j$. If these punctures are considered to be distinguishable, then the microstate count for the quantum $\mathrm{IH}$ resembles that of Maxwell-Boltzmann (MB) counting for a gas of distinguishable particles [3-6]. In this case, if the microcanonical entropy has to be given by Bekenstein-Hawking area law (BHAL) (i.e., one-fourth of the area of the horizon divided by Planck length squared), then the Barbero-Immirzi parameter $(\gamma)$ needs to take a certain fixed value.

However, recently there has been a trend of doing black hole thermodynamics in the quantum IH framework considering the punctures to be indistinguishable [7-10]. While in some cases Gibbs's approximation has been used in the $\mathrm{MB}$ counting to implement indistinguishability [10], the others use both Bose-Einstein (BE) and Fermi-Dirac (FD) statistics $[7,8]$ by treating the punctures literally as quantum mechanical particles with spins and thus categorizing the punctures with integral and half-integral spins into two different species which follow BE and FD statistics, respectively. Also, there is an instance where anyonic statistics have been used [9].

In this article, we shall revisit this issue of indistinguishability of punctures of quantum $\mathrm{IH}$. We assume that the punctures are indistinguishable. Consequently, the microstate count now resembles that of $\mathrm{BE}$ statistics applied to a gas of particles. We explain that the resemblance between a quantum $\mathrm{IH}$ and a gas of particles follows if we view the quantum number $j$ as denoting the area levels of an individual puncture, rather than "spin," similar to the energy levels of an individual particle in a gas. Automatically, this provides an explanation of how our work differs from other instances in literature where BE statistics have been discussed in relation to quantum $\mathrm{IH}$ by considering the quantum number $j$ as "spins" associated with the punctures (e.g., see [7]). Also, we explain why we think it is more reasonable to consider the quantum number $j$ as area levels of the individual punctures 
rather than "spin" analog of a particle by pointing out some fundamental difference between the physics associated with a quantum $\mathrm{IH}$ and a gas of particles, in spite of the structural similarity in their statistical mechanical framework. Then, we calculate the microcanonical entropy of an $\mathrm{IH}$ with a given classical area $A \gg \mathcal{O}\left(\ell_{p}^{2}\right)$ (where $\ell_{p}$ is the Planck length) and find the restrictions to be imposed on the BI parameter for the BHAL to follow. We find graphically that the BI parameter has an area dependence but approaches a constant value in the large area limit. Finally we end with some concluding remarks.

\section{Microstate Count}

An individual puncture of a quantum IH labeled with quantum number $j$ contributes a quantum of area $a_{j}$ to the quantum IH. If $s_{j}$ denotes the number of punctures with label $j$, then, for a set $\left\{s_{j}\right\}$, the total quantum area is given by $A_{\text {quant }}=\sum_{j} s_{j} a_{j}$. Also, $g_{j}$ is the degeneracy associated with a puncture labeled by quantum number $j$.

On the other hand, let us consider a gas of particles with total energy $\sum_{i} n_{i} \epsilon_{i}$, where $i \equiv$ single particle energy level (SPEL), $\epsilon_{i} \equiv$ energy of a particle in the $i$ th level, and $n_{i} \equiv$ number of particles in the $i$ th level. Let $\omega_{i}$ be the degeneracy associated with the $i$ th level.

There is a manifest structural similarity between these two systems if we consider the following correspondence $(\mathscr{G})$ :

$$
\begin{gathered}
j \longleftrightarrow i, \\
s_{j} \longleftrightarrow n_{i}, \\
a_{j} \longleftrightarrow \epsilon_{i}, \\
g_{j} \longleftrightarrow \omega_{i} .
\end{gathered}
$$

Thus, $j$ can be called single puncture area level (SPAL) for a quantum $\mathrm{IH}$.

As the underlying quantum theory of the gas of particles provides the details of $\epsilon_{i}, \omega_{i}$, and so forth, so does LQG for a quantum IH, namely, $a_{j}=8 \pi \gamma \ell_{p}^{2} \sqrt{j(j+1)}, g_{j}=(2 j+1)$, and $j$ can take values in the range $1 / 2,1,3 / 2, \ldots, A / 8 \pi \gamma \ell_{p}^{2}[1,2]$.

Now, for a quantum $\mathrm{IH}, s_{j}$ can vary arbitrarily without any restrictions and the punctures are indistinguishable by assumption. Hence, considering the correspondence $\mathscr{G}$, the system has a structural similarity with a gas of indistinguishable particles where $n_{i}$ can vary arbitrarily without any restrictions. The microstate count for a configuration $\left\{n_{i}\right\}$ is well known $[11,12]$. By the correspondence $\mathscr{G}$, we can simply get the number of microstates corresponding to a configuration $\left\{s_{j}\right\}$ of a quantum $\mathrm{IH}$, which is given by

$$
\Omega\left[\left\{s_{j}\right\}\right]=\prod_{j} \frac{\left(s_{j}+g_{j}-1\right) !}{s_{j} !\left(g_{j}-1\right) !} .
$$

(Digression. If we consider the punctures to be distinguishable and since any number of punctures can have any value of $j$, the microstate count for a set $\left\{s_{j}\right\}$ of punctures is given by $\Omega\left[\left\{s_{j}\right\}\right]=\left(\sum_{k} s_{k}\right) \prod_{j}\left(g_{j}^{s_{j}} / s_{j} !\right)[3,5,6]$, which resembles Maxwell-Boltzmann (MB) counting for a gas of particles considering the correspondence $\mathscr{G}[11,12]$.)

The counting details are available in standard textbooks of statistical mechanics (e.g., see $[11,12]$ ) and need not be discussed here unnecessarily. However, we shall cross-check the above formula with a simple example for a clarification. Let us consider that there are two punctures with $j=1 / 2$ and three punctures with $j=1$. So the corresponding degeneracies are $-1 / 2,1 / 2$ and $-1,0,1$, respectively. Since the punctures are indistinguishable, the distinct microstates that can be constructed out of the two $j=1 / 2$ punctures are $(1 / 2,1 / 2),(1 / 2,-1 / 2)$, and $(-1 / 2,-1 / 2)$. Similarly, for the three $j=1$ punctures the distinct microstates are $(1,1,1)$, $(1,1,0),(1,0,0),(0,0,0),(0,0,-1),(0,-1,-1),(-1,-1,-1)$, $(-1,-1,1),(-1,1,1)$, and $(-1,0,1)$. Hence, there are $3 \times 10=$ 30 distinct microstates of the system of five indistinguishable punctures. Now, if we put $s_{1 / 2}=2, g_{1 / 2}=2$ and $s_{1}=3, g_{1}=3$, then (2) yields 30 . So, the formula given by (2) stands justified.

This completes our effort to explain in what precise sense the punctures of a quantum $\mathrm{IH}$ obey $\mathrm{BE}$ statistics under the assumption of indistinguishability. However, we need to further clarify certain other issues regarding the analogy between a quantum IH and a gas of particles in order to differentiate our viewpoint from other instances in literature which discuss BE statistics in relation to quantum $\mathrm{IH}$.

From the correspondence $\mathscr{G}$ between a quantum $\mathrm{IH}$ and a gas of particles, it is manifest that there is no corresponding analog of spin of a particle for a puncture of quantum IH. The quantum number $j$ is called "spin" of a puncture in literature because it originates from the $\mathrm{SU}(2)$ spin representation carried by the edge of the bulk spin network graph that ends on the corresponding puncture $[1,2]$. But we have already explained that $j$ actually represents the SPAL of a quantum $\mathrm{IH}$ when it comes to microstate counting.

The spins of particles do not explicitly appear in the microstate counting. It only implicitly affects the counting by imposing restriction on $n_{i}$ through spin-statistics connection. On the other hand, any number of punctures can be associated with integral or half-integral $j$ - $s$ in case of quantum $\mathrm{IH}$. Alternatively, it can be said that there is no other independent quantum number (analog of particle spin) associated with the punctures of a quantum $\mathrm{IH}$ which imposes any restriction on $s_{j}$ implicitly.

In spite of all the above facts one can still wish to treat the quantum number $j$ as "spins" and punctures as particles. In that case, one needs to show how this treatment invokes the spin-statistics connection and affects the microstate count of a quantum IH. The main obstacle in taking this viewpoint is the deep mismatch between the fundamental natures of punctures of quantum $\mathrm{IH}$ and quantum mechanical particles. Particles are perturbative quantum excitations of matter fields propagating on smooth background geometry [13], whereas the punctures are nonperturbative background independent quantum geometric excitations of the IH $[1,2]$. The spinstatistics connection heavily relies on local Lorentz invariance related to the background spacetime. But, punctures of quantum $\mathrm{IH}$ being themselves the representatives of the 
quantum geometry, the notion of spin-statistics connection is hitherto unknown in this scenario.

\section{Entropy}

Now, we shall calculate the entropy of an IH with classical area $A \gg \mathcal{O}\left(\ell_{p}^{2}\right)$. The physical quantum states of the quantum $\mathrm{IH}$ which are of interest in this particular calculation are such that the quantum area corresponding to each state is within $\pm \mathcal{O}\left(\ell_{p}^{2}\right)$ window of the classical area $A$; that is, in mathematical language $A_{\text {quant }}\left[\left\{s_{j}\right\}\right]=A \pm \mathcal{O}\left(\ell_{p}^{2}\right)$. As we are working with $A \gg \mathcal{O}\left(\ell_{p}^{2}\right)$, we can neglect $\pm \mathcal{O}\left(\ell_{p}^{2}\right)$ for all practical purposes. Thus, the relevant configurations that will contribute to the entropy must obey the constraint

$$
C: 8 \pi \gamma \sum_{j} s_{j} \sqrt{j(j+1)}=\mathscr{A},
$$

where $\mathscr{A}=A / \ell_{p}^{2}$ is the dimensionless area of the quantum IH. So the entropy of the IH, using Boltzmann entropy formula and setting Boltzmann constant to unity, is then given by

$$
S=\log \left[\sum_{\left\{s_{j}\right\}} \Omega\left[\left\{s_{j}\right\}\right]\right],
$$

where the argument of the logarithm represents the total number of microstates arising from all possible configurations, the sum being over all possible configurations constrained by $C$. Assuming that the basic postulates of equilibrium statistical mechanics are valid in the present scenario of quantum IH, there is one most probable configuration whose corresponding number of microstates is overwhelmingly large compared to any other configuration $[11,12]$ such that the entropy of the $\mathrm{IH}$ can be approximately given by the entropy of the most probable configuration alone; that is,

$$
S=\log \left[\sum_{\left\{s_{j}\right\}} \Omega\left[\left\{s_{j}\right\}\right]\right]=\log \Omega\left[\left\{s_{j}^{\star}\right\}\right]
$$

+ contributions from the sub-dominant configurations

$$
\simeq \log \Omega\left[\left\{s_{j}^{\star}\right\}\right] \text {. }
$$

The most probable configuration can be obtained by extremizing the entropy corresponding to a configuration subject to the area constraint $C$ and hence it is the solution of the following equation:

$$
\delta \log \Omega\left[\left\{s_{j}\right\}\right]-\lambda \delta \mathscr{A}=0,
$$

where $\delta$ represents arbitrary variation with respect to the variable $s_{j}$ and $\lambda$ is the Lagrange multiplier. The distribution function for the most probable configuration (or the most probable distribution) comes out to be

$$
s_{j}^{\star}=\frac{\left(g_{j}-1\right)}{e^{8 \pi \lambda \gamma \sqrt{j(j+1)}}-1}=\frac{2 j}{e^{8 \pi \lambda \gamma \sqrt{j(j+1)}}-1} .
$$

Considering $s_{j} \gg 1$ and consequently applying Stirling's approximation, the entropy is calculated to be

$$
\begin{aligned}
S & \simeq \log \Omega\left[\left\{s_{j}^{\star}\right\}\right] \\
& =\lambda \mathscr{A}-\sum_{j=1 / 2}^{\mathscr{A} / 8 \pi \gamma} 2 j \log \left(1-e^{-8 \pi \lambda \gamma \sqrt{j(j+1)}}\right)+\sum_{j=1 / 2}^{\mathscr{A} / 8 \pi \gamma} \psi_{j},
\end{aligned}
$$

where the upper limit on $j$ is written in terms of $\mathscr{A}$ which is $j_{\max }=A / 8 \pi \gamma \ell_{p}^{2}=\mathscr{A} / 8 \pi \gamma$ and here

$$
\begin{aligned}
\psi_{j} & =\log \left(g_{j}-1\right) !-\left(g_{j}-1\right) \log \left(g_{j}-1\right)+\left(g_{j}-1\right) \\
& =\log (2 j) !-2 j \log (2 j)+2 j .
\end{aligned}
$$

The most probable distribution satisfies the area constraint which leads to the following equation:

$$
\begin{aligned}
\mathscr{A} & =8 \pi \gamma \sum_{j=1 / 2}^{\mathscr{A} / 8 \pi \gamma} \frac{\sqrt{j(j+1)}\left(g_{j}-1\right)}{e^{8 \pi \lambda \gamma \sqrt{j(j+1)}}-1} \\
& =16 \pi \gamma \sum_{j=1 / 2}^{\mathscr{A} / 8 \pi \gamma} \frac{j \sqrt{j(j+1)}}{e^{8 \pi \lambda \gamma \sqrt{j(j+1)}}-1} .
\end{aligned}
$$

Besides this, summing over $j$ gives the total number of punctures for the most probable distribution; that is,

$$
\begin{aligned}
N_{0} & :=\sum_{j} s_{j}^{\star}=\sum_{j=1 / 2}^{\mathscr{A} / 8 \pi \gamma} \frac{\left(g_{j}-1\right)}{e^{8 \pi \lambda \gamma \sqrt{j(j+1)}}-1} \\
& =\sum_{j=1 / 2}^{\mathscr{A} / 8 \pi \gamma} \frac{2 j}{e^{8 \pi \lambda \gamma \sqrt{j(j+1)}}-1} .
\end{aligned}
$$

It may be noted that the allowed values of $\lambda$ and $\gamma$ must be such that $N_{0} \gg 1$. This condition will be automatically satisfied if, at least, $\left.s_{j}^{\star}\right|_{\max } \gg 1$ is assured for the allowed values of $\lambda$ and $\gamma$. By $\left.s_{j}^{\star}\right|_{\max }$ we have meant the peak values of the curve $s_{j}^{\star}$ versus $j$ for given values of $\lambda$ and $\gamma$. As we proceed, we shall see that there exist such values of $\lambda$ and $\gamma$ for which the condition is satisfied.

Remarks. At this point, we would like to make a few general remarks. We have calculated the entropy of an IH with a given area and hence only the area constraint has appeared along with its corresponding Lagrange multiplier $\lambda$. However, it is unlike the calculations made in the author's one of the earlier publications [6]. The article [6] was meant to show that the entropy calculations, resulting in the area law and the subleading logarithmic terms, can also be done with fixed number of punctures directly from the Chern-Simons state counting formula of [14]. Also there was an attempt to give a reason why the number of punctures may be kept fixed for the calculations. All these were specifically meant to clarify the ad hoc proposal of fixing the number of punctures and calculating the entropy without making a connection to the 
physical Hilbert space of the quantum IH done in [4]. Hence, the article [6] has some issues of interest in its own right in the context of entropy calculation of quantum IH with fixed number of punctures and was particularly of interest in the contemporary flow of literature. However, in course of time, our understanding of quantum IH has evolved and we firmly believe that the fixation of the number of punctures may lead to new mathematical exercises, but the physically correct description of quantum IH does not allow one to a priori fix the number of punctures. This is because the full Hilbert space of an IH of a given area admits all possible number of punctures which can give rise to the quantum area within a Planck area window about the classical area $[1,2]$. Further, as we have already discussed in an earlier section, there is a structural similarity between the statistical mechanical framework of a quantum $\mathrm{IH}$ and a gas of particles if one considers a specific mapping. But this never implies that the punctures can be literally treated as particles. Indeed, the very notion of background independent approach of LQG differentiates the notion of punctures from the notion of particles arising from background dependent quantum field theory. Unlike what occurs in case of quantum field theory one can develop a notion of particle number from the number operator and, by showing it as a conserved quantity, there is no such construction in existence for the number of punctures of quantum IH in LQG framework. We hope these arguments clarify our shift in paradigm towards keeping the number of punctures arbitrary in case of entropy calculation of quantum IH.

\section{The BHAL and the Barbero-Immirzi Parameter}

The next step is to choose $\gamma$ in such a way that (8) yields the BHAL. Hence, we demand that the entropy should be given by the BHAL; that is,

$$
S=\frac{\mathscr{A}}{4}
$$

and then explore the consequences. Now, demanding (12) we obtain the following equation:

$$
\lambda \mathscr{A}-\sum_{j=1 / 2}^{\mathscr{A} / 8 \pi \gamma} 2 j \log \left(1-e^{-8 \pi \lambda \gamma \sqrt{j(j+1)}}\right)+\sum_{j=1 / 2}^{\mathscr{A} / 8 \pi \gamma} \psi_{j}=\frac{\mathscr{A}}{4} .
$$

Our motto is to show that there exists certain value(s) of $\gamma$ for which the BHAL can be derived from the indistinguishable punctures, which is equivalent to finding the solutions of (10) and (13). This can be accomplished by finding if there is any intersection of the $2 \mathrm{D}$ surfaces described by (10) and (13) in the $3 \mathrm{D}$ space spanned by $\mathscr{A}, \lambda$, and $\gamma$. Such a plot in Mathematica, shown in Figure 1, reveals that there indeed exist values of $\gamma$, which furthermore fulfill certain relevant criteria as follows:

(i) $\gamma$ should lie in the range $0<\gamma \leq \mathscr{A} / 4 \pi$ because $j_{\max }=$ $\mathscr{A} / 8 \pi \gamma \geq 1 / 2$ and $\gamma$ appears in the area spectrum as a multiplicative factor and hence needs to be positive definite.

(ii) The allowed values of $\gamma$ are such that we have $\mathscr{A} \gg$ $\mathcal{O}(1)$ and $\lambda>0$ for $s_{j}^{\star}$ to be positive definite for all $j$ (see (7)).

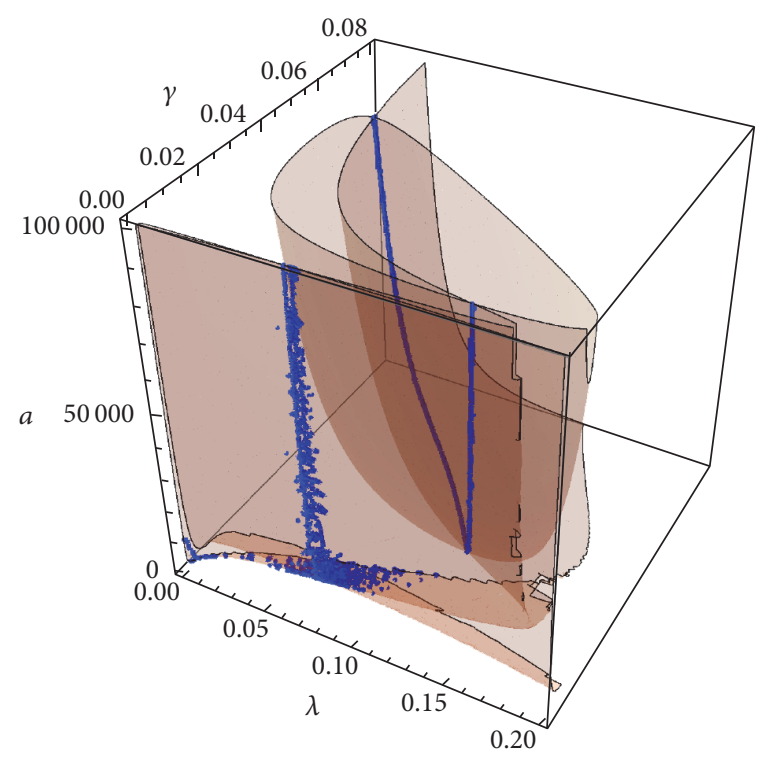

FIgure 1: The blue curves are the solutions of (10) and (13). $\mathscr{A}$ is symbolized as " $a$ " in the 3D plot. There are two branches of solutions, of which the U-shaped curve is excluded on physical grounds. The other curve is the only feasible solution which has a broadening due to graphical impreciseness of the plot. (See the Appendix.)

On physical grounds we shall exclude the U-shaped curve from consideration because it gives rise to multiple values of $\gamma$ for a given $\mathscr{A}$ which imply the following unphysical result: for a given $\mathscr{A}$ we will have two different most probable spin distributions. So we are left with the other curve which will give us the value(s) of $\gamma$ for which the BHAL is valid. From Figure 2, which gives us the view of $3 \mathrm{D}$ plot along the $\lambda$-axis, we see that with increasing area $(\mathscr{A})$ the curve straightens very rapidly and asymptotically approaches to some constant value $\gamma_{0}$ (e.g.). Besides that, from Figure 1 it was already evident that $\lambda$ becomes independent of $\mathscr{A}$ with increasing $\mathscr{A}$. Hence, for $\mathscr{A} \gg \mathscr{O}(1)$, we have practically $\gamma=\gamma_{0}$ and $\lambda=\lambda_{0}$ for the BHAL to hold. In view of this, we can conclude that the BHAL follows from the LQG description of black hole horizon, with the same consequences whether we consider the punctures to be distinguishable or not. The only difference is the value of $\gamma_{0}$ which is of the order of $10^{-3}$ here but of the order of $10^{-1}$ in case of distinguishable punctures. It may be further noted that the order of magnitude of $\gamma$ and $\lambda$ along the curve ensures that at least $\left.s_{j}^{\star}\right|_{\max } \gg 1$ so that Stirling's approximation is valid. One can check that to remain assured.

\section{State Counting of Quantum SU(2) Chern-Simons Theory Coupled to Indistinguishable Sources: Some Speculations}

In this section we discuss a few possible new ideas which germinate from this exercise of state counting and entropy calculation for quantum $\mathrm{IH}$ with indistinguishable punctures. To begin with, we briefly discuss the subject matter of [6]. In [6], 


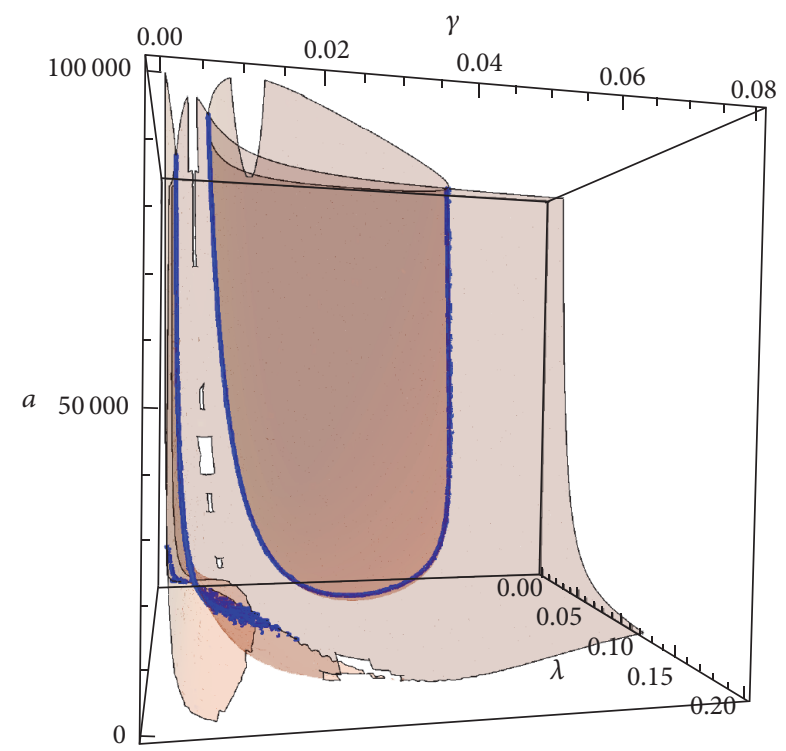

FIgURE 2: The view of the plot in Figure 1 along the $\lambda$-axis reveals the variation of $\gamma$ with $\mathscr{A}$ (symbolized as $a$ in the figure). As $\mathscr{A}$ increases, the value of $\gamma$ asymptotically approaches a fixed value $\gamma_{0}$ (e.g.) of the order of $10^{-3}$. (See the Appendix.)

we begin with the original formula of state count for a set of $N$ punctures with spins $\left(j_{1}, j_{2}, \ldots, j_{N}\right)[14]$. Then we do a sum over all possible spins and using multinomial expansion switch over to the expression of microstates in terms of the spin configurations. Then, entropy of an $\mathrm{IH}$ with given area is calculated by the method of most probable distribution for fixed number of punctures (and for arbitrary number of punctures see [15]). That the punctures were considered to be distinguishable during state counting becomes explicit when we switch over to the spin configuration language. The expression for the number of microstates for a given spin configuration $\left\{s_{j}\right\}$ is given by [6]

$$
\Omega_{\text {dist. }}\left[\left\{s_{j}\right\}\right]=\frac{2}{k+2} \sum_{a=1}^{k+1} \sin ^{2} \frac{a \pi}{k+2} \underbrace{\frac{\left(\sum_{l} s_{l}\right)}{\prod_{j} s_{j} !} \prod_{j} g_{j}^{s_{j}}(a, k)}_{\text {MB statistics by } \mathscr{G}},
$$

where $g_{j}(a, k)=\{\sin (((2 j+1) a \pi) /(k+2)) / \sin ((a \pi) /(k+$ $2))\}$. The underbraced part clearly resembles the MB state counting for ideal gas if we consider the correspondence $\mathscr{G}$ and manifests the distinguishability of the punctures through the combinatorial expression. In fact, in the large $k$ limit, converting the sum over $a$ into an integration over a continuous variable, say $\theta$, followed by a saddle point approximation about a value $\theta=\theta_{0}$, expression (14) reduces to

$$
\Omega_{\text {dist. }}\left[\left\{s_{j}\right\}\right] \simeq \frac{\left(\sum_{l} s_{l}\right) !}{\prod_{j} s_{j} !} \prod_{j} g_{j}^{s_{j}},
$$

with $g_{j}=2 j+1$, as the zeroth-order (in the fluctuations about $\theta_{0}$ ) term. This formula is nothing but the state count for distinguishable punctures with configuration $\left\{s_{j}\right\}$, obtained by ignoring the deeper details of the state counting associated with the internal symmetry of the quantum IH. It is the effective formula which leads to the leading term of the entropy which is proportional to area $[6,15]$. Interestingly, it may be noted that the combinatorics involved in formula (15) is present in the exact form in the underbraced part of formula (14), except the fact that $g_{j}$ differs.

Now, when we consider the punctures to be indistinguishable, we do not have a complete formula for the microstate count from which such a similar exercise can be performed. So, we try to go in the reverse direction in this case and look for the possible complete formula for the state count which takes into account the underlying symmetry of the $\mathrm{IH}$ and the associated Chern-Simons theory. Thus, the first step for the state counting for indistinguishable punctures is to ignore the minute details involved with the symmetries and so forth of the quantum $\mathrm{IH}$ and do the state counting for a configuration $\left\{s_{j}\right\}$ of indistinguishable punctures and this is what has resulted in formula (2). Further, we have also shown that this state count results in the BHAL, that is, the leading term of the entropy.

Henceforth, taking cue from the case of distinguishable punctures, we speculate that the combinatorial form of formula (2) for indistinguishable punctures should appear in the complete formula which takes into account the underlying symmetry of the quantum $\mathrm{IH}$ and the associated ChernSimons theory, as follows:

$$
\begin{aligned}
& \Omega_{\text {indist. }}\left[\left\{s_{j}\right\}\right] \\
& =\frac{2}{k+2} \sum_{a=1}^{k+1} \sin ^{2} \frac{a \pi}{k+2} \underbrace{\prod_{j} \frac{\left(s_{j}+g_{j}(a, k)-1\right) !}{s_{j} !\left(g_{j}(a, k)-1\right) !}}_{\text {BE statistics by } \mathscr{G}},
\end{aligned}
$$

except the fact that the form of $g_{j}$ differs. This above formula is what we expect to get while we do the state counting for the quantized SU(2) Chern-Simons theory, in the large $k$ (weak coupling) limit, coupled to a configuration $\left\{s_{j}\right\}$ of indistinguishable sources. Although this is only a guess, it gives us a hint towards what the answer may look like in case of indistinguishable punctures when it will be derived from counting the dimensionality of the Hilbert space associated with SU(2) Chern-Simons theory coupled to indistinguishable sources like what was done for distinguishable sources in [14]. Further, if the above formula can be shown to be the correct and the complete one, then there may be a way to find some approximation to get a correction to formula (2) to obtain the subleading correction to the BHAL.

Apart from this, the problem becomes more interesting due to the fact that it has a direct link with the counting of the conformal blocks of Wess-Zumino-Witten model [16]. This may lead to some new physical problems in the context of conformal quantum field theories. Thus, the exercise presented here, which invokes the idea about how to implement the statistics in case of indistinguishable punctures of quantum IH correctly, may be considered as a small but crucial first step towards exploring potential new physics problems related to quantum $\mathrm{IH}$ with indistinguishable punctures and beyond. 


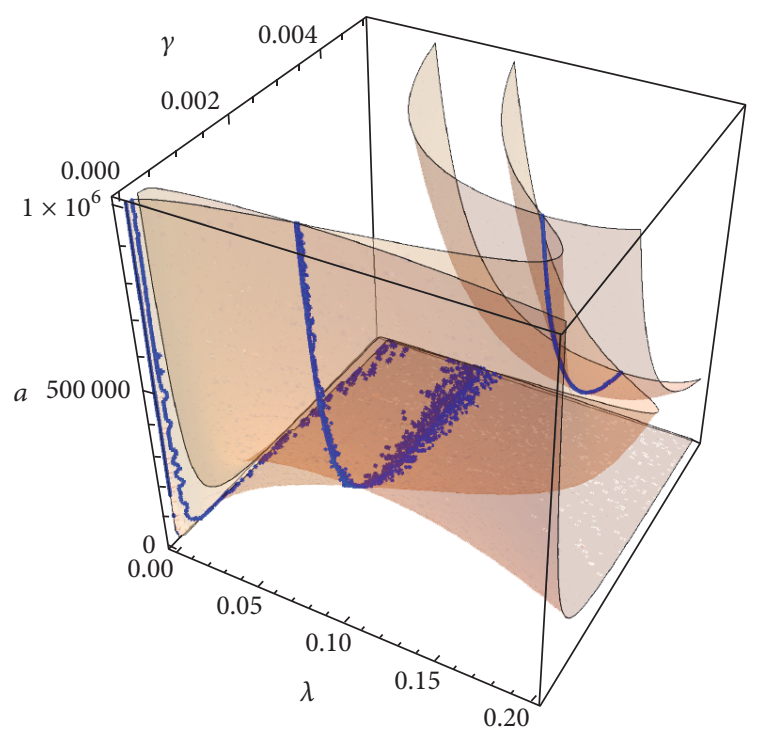

FIgURE 3: This is a magnified view of the plot in Figure 1 to focus on the curve which gives the solution (along midway of the $\lambda$-axis).

\section{Conclusion}

What we have done here is the entropy calculation of the $\mathrm{IH}$ by assuming that the punctures of quantum $\mathrm{IH}$ are indistinguishable and we do not go into the debate whether this assumption is justified within this quantum IH framework. As far as the physical result is concerned, that is, the BHAL resulting from an $a b$ initio statistical mechanical calculation from the quantum geometry of black hole horizon as laid down by LQG, there is no much difference whether we consider the punctures to be distinguishable or not. Given that the calculations are done for large area black holes, the BHAL is obtained for a constant value of $\gamma$, except the fact that order of magnitude of $\gamma$ is different in case of distinguishable and indistinguishable punctures.

However, there is a very important issue which we are unable to address here in the context of the assumption of indistinguishability. The states of the quantum IH are actually given by that of the quantum $\mathrm{SU}(2)$ Chern-Simons theory coupled to the punctures [14]. Now, in the case of distinguishable punctures, it can be shown that the MB counting results from a zeroth-order approximation of the complete formula for the state counting [6]. The distinguishability is inherited by the fact that we consider a sum over all values of spins on the microstate count for a given set of spins $j_{1}, \ldots, j_{N}$ and arrive at the $\mathrm{MB}$ counting for a given spin configuration by the application of multinomial expansion [6]. The next order correction leads to the logarithmic correction $[6,15]$. Now, when the punctures are indistinguishable, we can not carry out a naive sum over all values of spins for a given puncture data as this will count the microstates which are meant to be indistinguishable. Thus, under the assumption of indistinguishability of punctures, it will be an interesting problem to look upon how the BE counting formula, as discussed here, comes out as an approximation of the exact scenario of quantum Chern-Simons theory. Then it will also

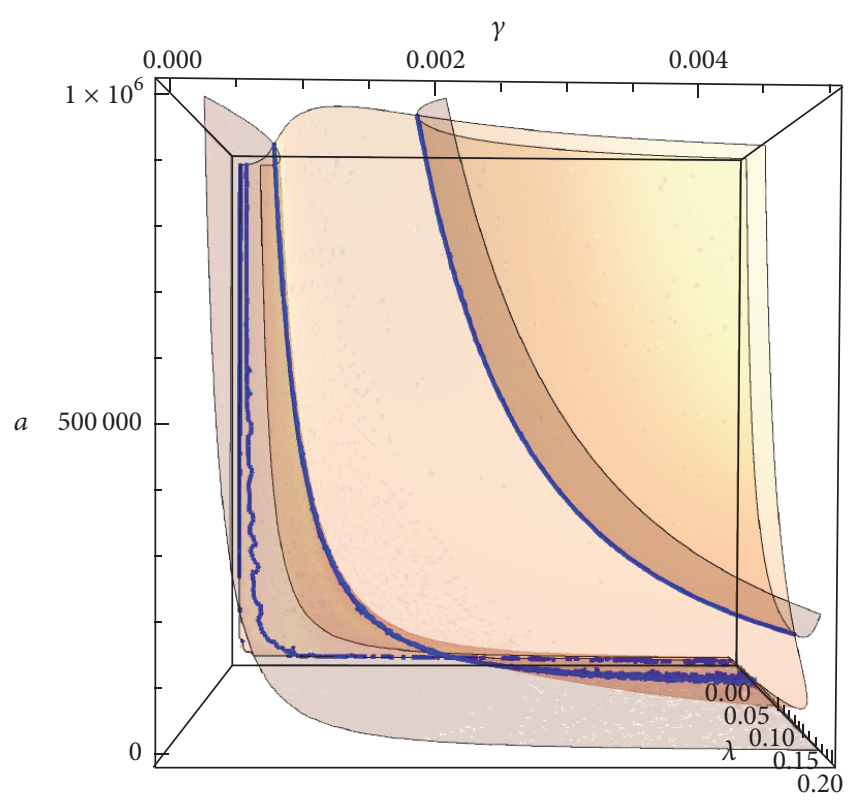

FIgURE 4: This is a magnified view of the plot in Figure 2 to show the view along $\lambda$-axis more closely.

be clear what the next order correction is. Will it be the same known logarithmic correction $-3 / 2 \log \mathscr{A}$ [17]? We hope to investigate this problem in future.

\section{Appendix}

Here we have presented some magnified versions of the $3 \mathrm{D}$ plot in Figures 3 and 4.

\section{Competing Interests}

The author declares that there is no conflict of interests regarding the publication of this paper.

\section{Acknowledgments}

The author thanks Romesh Kaul for discussions regarding a few issues of this work. This work is funded by The Department of Atomic Energy, India.

\section{References}

[1] A. Ashtekar, J. Baez, A. Corichi, and K. Krasnov, "Quantum geometry and black hole entropy," Physical Review Letters, vol. 80, no. 5, pp. 904-907, 1998.

[2] A. Ashtekar, J. Baez, and K. Krasnov, "Quantum geometry of isolated horizons and black hole entropy," https://arxiv.org/ abs/gr-qc/0005126.

[3] A. Ghosh and P. Mitra, "An improved estimate of black hole entropy in the quantum geometry approach," Physics Letters $B$, vol. 616, no. 1-2, pp. 114-117, 2005.

[4] A. Ghosh and A. Perez, "Black hole entropy and isolated horizons thermodynamics," Physical Review Letters, vol. 107, no. 24, Article ID 241301, 5 pages, 2011. 
[5] A. Majhi, "The microcanonical entropy of quantum isolated horizon, 'quantum hair' $N$ and the Barbero-Immirzi parameter fixation," Classical and Quantum Gravity, vol. 31, no. 9, Article ID 095002, 2014.

[6] A. Majhi and P. Majumdar, "Quantum hairs' and entropy of the quantum isolated horizon from Chern-Simons theory," Classical and Quantum Gravity, vol. 31, no. 19, Article ID 195003, 2014.

[7] A. Ghosh, K. Noui, and A. Perez, "Statistics, holography, and black hole entropy in loop quantum gravity," Physical Review D, vol. 89, no. 8, Article ID 084069, 12 pages, 2014.

[8] O. Asin, J. B. Achour, M. Geiller, K. Noui, and A. Perez, "Black holes as gases of punctures with a chemical potential: Bose-Einstein condensation and logarithmic corrections to the entropy," Physical Review D, vol. 91, no. 8, Article ID 084005, 15 pages, 2015.

[9] A. G. A. Pithis and H.-C. R. Euler, "Anyonic statistics and large horizon diffeomorphisms for loop quantum gravity black holes," Physical Review D, vol. 91, no. 6, Article ID 064053, 22 pages, 2015.

[10] M. Han, "Black hole entropy in loop quantum gravity, analytic continuation, and dual holography," https://arxiv .org/abs/1402.2084.

[11] R. K. Pathria and P. D. Beale, Statistical Mechanics, Elsevier, 2011.

[12] K. Huang, Statistical Mechanics, 2, 3, John Wiley \& Sons, 1987.

[13] M. E. Peskin and D. V. Schroeder, An Introduction to Quantum Field Theory, 1995.

[14] R. K. Kaul and P. Majumdar, "Quantum black hole entropy," Physics Letters. B, vol. 439, no. 3-4, pp. 267-270, 1998.

[15] R. K. Kaul, "Entropy of quantum black holes," Symmetry, Integrability and Geometry: Methods and Applications (SIGMA), vol. 8, article 005, 30 pages, 2012.

[16] E. Witten, "Quantum field theory and the Jones polynomial," Communications in Mathematical Physics, vol. 121, no. 3, pp. 351399, 1989.

[17] R. K. Kaul and P. Majumdar, "Logarithmic correction to the Bekenstein-Hawking entropy," Physical Review Letters, vol. 84, no. 23 , p. $5255,2000$. 

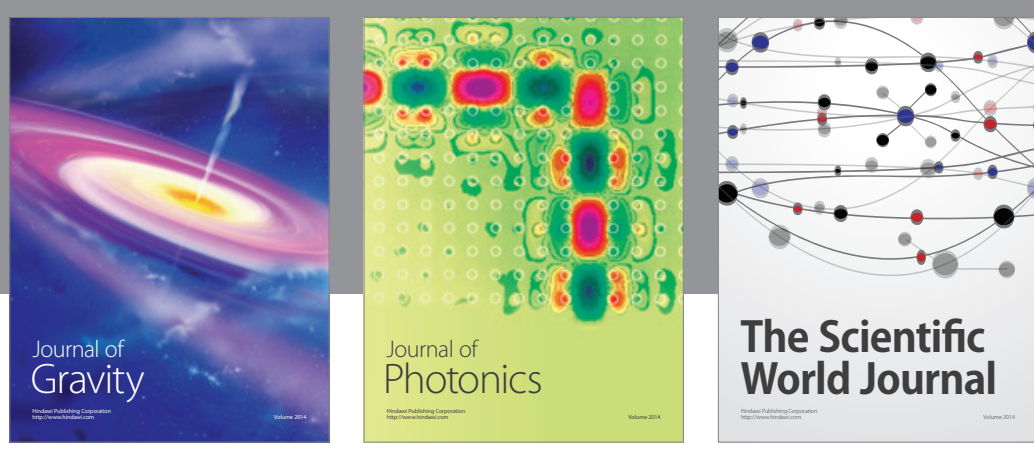

The Scientific World Journal
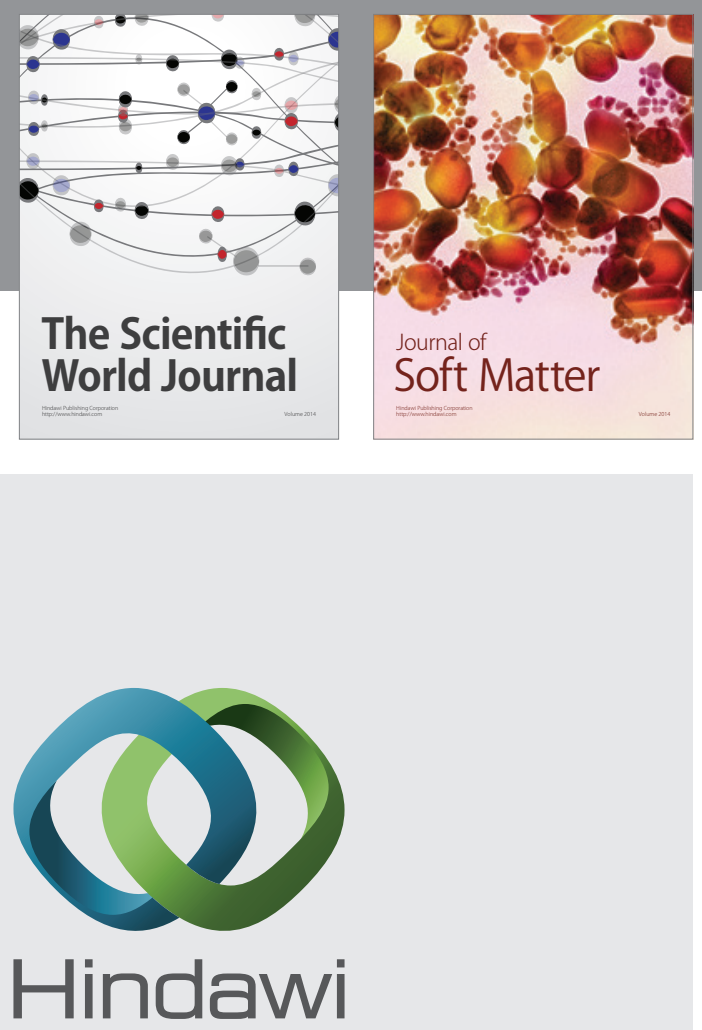

Submit your manuscripts at

http://www.hindawi.com

nternational Journal of

Statistical Mechanics
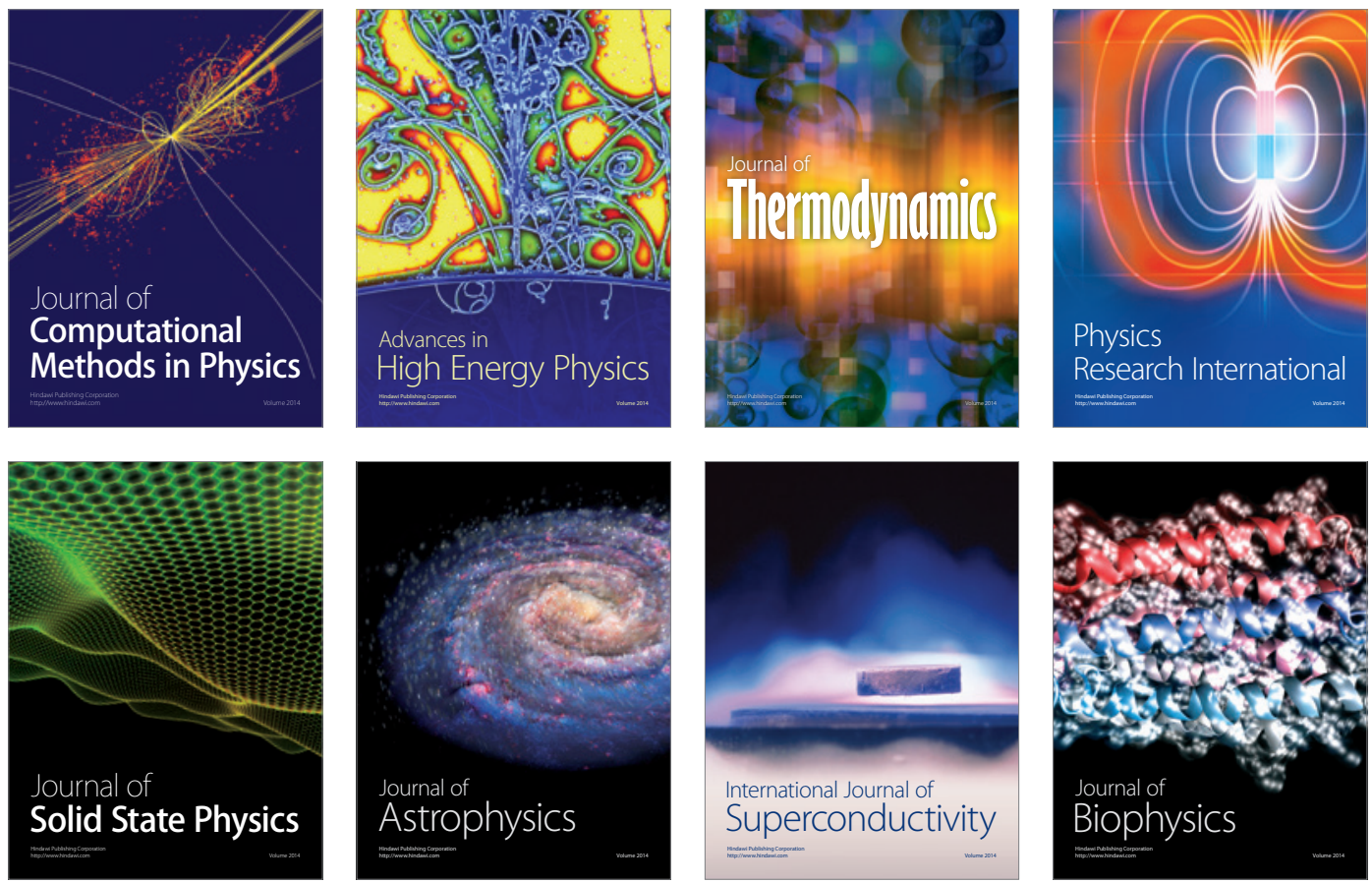
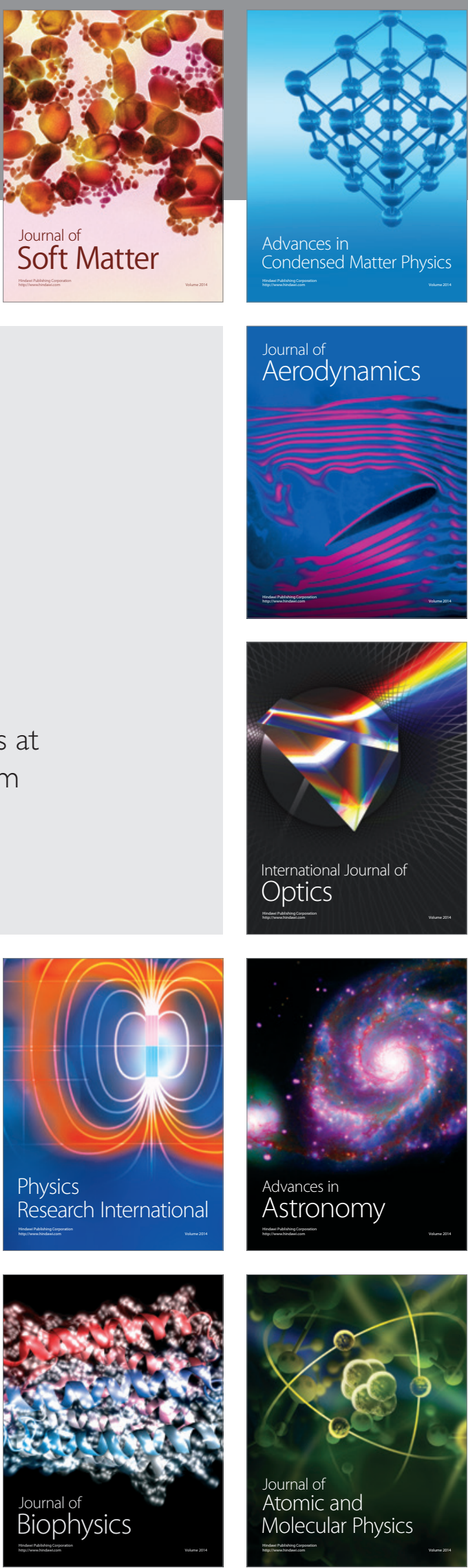